\title{
An extension of the disc Algebra
}

\author{
V. Nestoridis
}

\begin{abstract}
We identify all uniform limits of polynomials on the closed unit disc $\bar{D}$ with respect to the chordal metric $\chi$ on $\mathbb{C} \cup\{\infty\}$. One such limit is $f \equiv \infty$. The other limits are holomorphic functions $f: D \rightarrow \mathbb{C}$ so that for every $\zeta \in \partial D$ the $\lim _{\substack{z \rightarrow \zeta \\ z \in D}} f(z)$ exists in $\mathbb{C} \cup\{\infty\}$. The class of the above functions is denoted by $\widetilde{A}(D)$. We study properties of the members of $\widetilde{A}(D)$, as well as, some topological properties of $\widetilde{A}(D)$ endowed with its natural metric topology. There are several open questions and new directions of investigation.
\end{abstract}

AMS classification number: Primary 30J99, secondary 46A99, 30E10

Key words and phrases: Disc Algebra, Mergelyan's Theorem, chordal metric, polynomial approximation, generic property.

\section{Introduction}

In this article we consider $\chi$ the chordal metric in $\mathbb{C} \cup\{\infty\}$, where $\mathbb{C}$ denotes the complex plane. First we investigate the set of uniform limits on the closed unit disc $\bar{D}$ of the polynomials with respect to the metric $\chi$. One such limit is $f \equiv \infty$. The other limits are exactly functions $f: D \rightarrow C$ holomorphic in the open unit disc $D$, such that for every $\zeta \in T=\partial D$ the limit $\lim _{\substack{z \rightarrow \zeta \\ z \in D}} f(z)$ exists in $\mathbb{C} \cup\{\infty\}$. The class of the above functions is an extension of the disc algebra $A(D)$ and is denoted by $\widetilde{A}(D)$.

If $f \in \widetilde{A}(D)$ is such that $f \not \equiv \infty$, then Privalov's Theorem implies that $f^{-1}(\infty)$ is a compact subset of the unit circle $T$ with zero length. Reversely for every such set, there exist $f \in \widetilde{A}(D), f \not \equiv \infty$ so that $f^{-1}(\infty)$ is exactly this set. Compact subsets of $T$ with positive length are not compact of interpolation for $\widetilde{A}(D)$. It is an open question if every compact subset $E \subset T$ with zero length is a compact of interpolation for $\widetilde{A}(D)$. That is, is it true that every continuous function $h: E \rightarrow \mathbb{C} \cup\{\infty\}$ has an extension in $\widetilde{A}(D)$. 
For general $f, g \in \widetilde{A}(D)$ it is not true that $\sup _{z \in \bar{D}} \chi(f(z), g(z)) \leq \sup _{|z|=1} \chi(f(z), g(z))$, even if we multiply the right member by a constant $C<+\infty$ independent of $f, g$. However two members of $\widetilde{A}(D)$ which concide on $T$, should be identical. The mean value property fails in general in $\widetilde{A}(D)$.

The disc algebra $A(D)$ endowed with the supremum norm is a Banach algebra, so in particular a complete metric space. The larger class $\widetilde{A}(D)$ is naturally endowed with the metric $\widetilde{d}(f, g)=\max _{|z| \leq 1} \chi(f(z), g(z)), f, g \in \widetilde{A}(D)$. The metric space $(\widetilde{A}, \widetilde{d})$ is also complete. Often we write $\chi(f, g)$ for $\widetilde{d}(f, g), f, g \in \widetilde{A}(D)$. Then $A(D)$ is an open dense subset of $\widetilde{A}(D)$. Furthermore the relative topology of $A(D)$ from $\widetilde{A}(D)$ coincides with the usual topology of $A(D)$. For $f \in \widetilde{A}(D)$ we denote by $E_{f}$ the set $E_{f}=\{\zeta \in T: f(\zeta) \notin f(D)\}$. We prove that generically, for every $f \in \widetilde{A}(D)$ the set $E_{f}$ has zero length. Also we show that the set $Y=\{f \in \widetilde{A}(D): f(D) \subset f(T)\}$ is a non-void closed subset of $\widetilde{A}(D)$ of first category. We also introduce the set $W=\{f \in$ $\widetilde{A}(D): f(T)=\mathbb{C} \cup\{\infty\}\}$. This is a closed subset of $\widetilde{A}(D)$ of first category, but we do not know if it is non-empty. If we assume that every compact subset of $T$ with zero length is a compact of interpolation for $\widetilde{A}(D)$, then we can show that $W$ is non-empty.

Another open question is to characterize the compact subsets of $\bar{D}$ which are of interpolation for $\widetilde{A}(D)$. A necessary condition is that, they are of the form $E \cup\left\{z_{n}\right.$ : $n \in I\}, I$ finite or infinite denumerable, $\left|z_{n}\right|<1$ for all $n \in I$, where $E \subset T$ is compact with zero length and all limit points of $z_{n}, n \in I$ are included in $E$. Is the same condition sufficient or not? If not, what is a nessary and sufficient condition? Another open question is to characterize the zero sets in $\bar{D}$ of the members of $\widetilde{A}(D)$. Every result which holds in $A(D)$ may be examined if it holds in $\widetilde{A}(D)$.

In the above discussion the uniform convergence with respect to the metric $\chi$ was considered mainly on $\bar{D}$, but we can consider it on other compact subsets of $\mathbb{C}$. For instance, does Mergelyan's Theorem holds for this uniform convergence with respect to the metric $\chi$ ? In general, this is an open question:

Question: Let $L \subset \mathbb{C}$ be a compact set with connected complement. Let $f: L \rightarrow$ $\mathbb{C} \cup\{\infty\}$ be a continuous function such that for every component $V$ of $L^{0}$ either $f_{\mid V} \equiv \infty$ or $f(V) \subset \mathbb{C}$ and $f_{\mid V}$ is holomorphic. Is then true that there exists a sequence of polynomials converging to $f$ uniformly on $L$ with respect to the metric $\chi$ ?

It is easy to see that the converse holds. 
In the present article we give a positive answer to the previous question in a few particular cases, while the general case is open.

More generally one can investigate the uniform limits with respect to the metric $\chi$ on other sets, as an annulus or a circle, of a sequence of rational functions with prescribed set of poles. For instance I do not know what are the limits on a circle when we approximate by polynomials. On the contrary on the unit circle, if we approximate with trigonometric polynomials, the limits are all continuous functions $f: T \rightarrow \mathbb{C} \cup\{\infty\}$.

Therefore, we see that there are several directions to continue our investigation.

Before closing we say that, if we consider $H(D)$, the space of holomorphic functions in $D$, endowed with the topology of uniform convergence on compacta, then $\widetilde{A}(D) \cap$ $H(D)$ is a subset of $H(D)$ of first category. One reason is that $\widetilde{A}(D)$ is disjoint with the residual set $U$ in $H(D)$ of universal Taylor series ([1, [2]). In fact if $f \in U$, then it is impossible to have a finite (in $\mathbb{C}$ ) limit $\lim _{z \rightarrow e^{i \vartheta \vartheta}} f(z)([3])$.

\section{The definition}

We consider the open unit disc $D=\{z \in \mathbb{C}:|z|<1\}$ in the complex plane $\mathbb{C}$ and we fixe $w \in \mathbb{C} \cup\{\infty\}$. We are looking for the usual uniform limits on the closed unit disc $\bar{D}=\{z \in \mathbb{C}:|z| \leq\}$ of rational functions whose poles in $\mathbb{C} \cup\{\infty\}$ are included in $\{w\}$. For $w=\infty$ we are looking for the usual uniform limits on $\bar{D}$ of polynomials $P(z)=\sum_{n=0}^{N} a_{n} z^{n}, a_{n} \in \mathbb{C}, N=0,1,2, \ldots$. It is well known that the set of such limits coincides with the disc Algebra $A(D)=\{f: \bar{D} \rightarrow \mathbb{C}$, continuous on $\bar{D}$ and holomorphic in $D\}$. If $w \in \mathbb{C},|w|>1$ we are looking for the usual uniform limits

on $\bar{D}$ of functions of the form $P(z)=\sum_{n=0}^{N} a_{n} \frac{1}{(z-w)^{n}}, a_{n} \in \mathbb{C}, N=0,1,2, \ldots$. Then the set of such limits ia again the disc Algebra $A(D)$.

If $w \in \mathbb{C},|w| \leq 1$, the function $P(z)=f(z)$ satisfies $f(w)=\infty$, with $w \in \bar{D}$ or $f$ is constant. Looking for uniform limits with respect to the Eucledian metric in $\mathbb{C} \cong \mathbb{R}^{2}$ we exclude the cases $f(w)=\infty$, so the set of such limits should be the set of constant functions $g(z)=c$ for all $z \in \bar{D}$ with $c \in \mathbb{C}$. The answer is not satisfactory, because the set of limits does not contain the trivial cases of constant sequences as $f_{n}(z)=\frac{1}{z-w}$, $n=1,2, \ldots$. Such a sequence should converge to the function $f(z)=\frac{1}{z-w}$; but, this is excluded, because it takes the value $\infty$ in $z=w \in \bar{D}$. This leads us to search for the uniform limits of the rational functions with poles in $\mathbb{C} \cup\{\infty\}$ included in $\{w\}$ with 
respect to the chordal distance $\chi$ on $\mathbb{C} \cup\{\infty\}$.

We have

$$
\begin{aligned}
\chi\left(z_{1}, z_{2}\right) & =\frac{\left|z_{1}-z_{2}\right|}{\sqrt{1+\left|z_{1}\right|^{2}} \sqrt{1+\left|z_{2}\right|^{2}}} \text { for } z_{1}, z_{2} \in \mathbb{C} \\
\text { and } \chi(z, \infty) & =\frac{1}{\sqrt{1+|z|^{2}}} \text { for } z \in \mathbb{C} \text { and } \chi(\infty, \infty)=0 .
\end{aligned}
$$

Geometrically, we identify $\mathbb{C} \cup\{\infty\}$ with $S^{2} \subset \mathbb{R}^{3}$ via stereographic projection and $\chi$ is a constant multiple of the restriction on $S^{2}$ of the usual Eucledian metric in $\mathbb{R}^{3}$.

If $f_{n}, f: E \rightarrow \mathbb{C} \cup\{\infty\}, n=1,2, \ldots$ are functions defined on a set $E$, then $f_{n} \rightarrow f$ uniformly on $E$, with respect to the metric $\chi$ if and only if, $\sup _{z \in E} \chi\left(f_{n}(z), f(z)\right) \rightarrow 0$, as $n \rightarrow+\infty$. With this convergence in mind, we must reexamine the cases $w=\infty$ or $w \in \mathbb{C},|w|>1$, as well.

Suppose $w=\infty$. Assume that a sequence of polynomials $f_{n} n=1,2, \ldots$ converges to a function $f: \bar{D} \rightarrow \mathbb{C} \cup\{\infty\}$ uniformly on $\bar{D}$ with respect to $\chi$. Since uniform convergence preserves continuity, it follows that $f$ is continuous when $\mathbb{C} \cup\{\infty\}$ is endowed with the metric $\chi$. Suppose that $f\left(z_{0}\right)=\infty$ for some $z_{0},\left|z_{0}\right|<1$. Then for some $r>0$ small we have $\chi\left(f\left(z_{0}\right)=\infty, f(z)\right)<\frac{1}{3}$ for all $z:\left|z-z_{0}\right|<r$. Since $f_{n} \rightarrow f$ uniformly with respect to $\chi$, there exists $n_{0}$ so that $\chi\left(f_{n}(z), \infty\right)<\frac{1}{2}$ for all $n \geq n_{0}$ and all $z:\left|z-z_{0}\right|<r$. Thus, $f_{n}(z) \in \mathbb{C}-\{0\}$ for all $n \geq n_{0}$ and $z:\left|z-z_{0}\right|<r$. Since $f_{n}$ is holomorphic in $\mathbb{C}$, being a polynomial, it follows that $\frac{1}{f_{n}}$ is holomorphic in the $\operatorname{disc}\left\{z:\left|z-z_{0}\right|<r\right\}$. As $\chi\left(\frac{1}{z_{1}}, \frac{1}{z_{2}}\right)=\chi\left(z_{1}, z_{2}\right)$ for all $z_{1}, z_{2} \in \mathbb{C} \cup\{\infty\}$, it follows that $\frac{1}{f_{n}} \rightarrow \frac{1}{f}$ uniformly on $\left\{z:\left|z-z_{0}\right|<r\right\}$ with respect to the distance $\chi$. Since $\chi(\infty, f(z))<\frac{1}{3}$ it follows $\chi\left(0, \frac{1}{f(z)}\right)<\frac{1}{3}$ for all $z:\left|z-z_{0}\right|<r$ and $\chi\left(0, \frac{1}{f_{n}(z)}\right)<\frac{1}{2}$ for all $z:\left|z-z_{0}\right|<4$ and $n \geq n_{1}$, for some $n_{1} \geq n_{0}$. Thus, there exist $M<+\infty$ so that $\left|\frac{1}{f_{n}(z)}\right|<M$ and $\left|\frac{1}{f(z)}\right|<M$ for all $n \geq n_{1}$ and $z:\left|z-z_{0}\right|<r$. It follows easily

$\sup _{z:\left|z-z_{0}\right|<r}\left|\frac{1}{f_{n}(z)}-\frac{1}{f(z)}\right| \leq \sup _{z:\left|z-z_{0}\right|<r} \chi\left(\frac{1}{f_{n}(z)}, \frac{1}{f(z)}\right) \cdot C$ for all $n \geq n_{1}$ with $C<+\infty$.

Thus, the sequence of holomorphic mappings $\frac{1}{f_{n}}, n \geq 1$ converges uniformly on $\{z$ : $\left.\left|z-z_{0}\right|<r\right\}$ to $\frac{1}{f}$ with respect to the usual Eucledian distance on $\mathbb{C}=\mathbb{R}^{2}$. But $\frac{1}{f_{n}(z)} \neq 0$ for all $z:\left|z-z_{0}\right|<r, n \geq n_{1}$ and $\frac{1}{f\left(z_{0}\right)}=0$. Hurwitz Theorem implies $f \equiv \infty$ on $\left\{z:\left|z-z_{0}\right|<r\right\}$. Thus the set $f^{-1}(\infty) \cap D$ is open. The same set is relatively closed by continuity. Since $D$ is connected, it follows $f \equiv \infty$. Reversely, for $P_{n}(z) \equiv n$ the limit is $\infty$. So the constant infinity function is one possible limit. If $f$ is not identically 
equal to $\infty$ and $f$ is a possible limit, then $f(D) \subset \mathbb{C}$ and the value $\infty$ is possible only on the boundary $\partial D=T$ where $T=\{z \in \mathbb{C}:|z|=1\}$ is the unit circle. It is also easily seen, by the previous discussion, that $f_{n} \rightarrow f$ uniformly on compact subsets of $D$ with respect to the usual Eucledian metric in $\mathbb{C}=\mathbb{R}^{2}$. Thus, $f$ is holomorphic in $D$.

Theorem 2.1. Let $f: D \rightarrow \mathbb{C} \cup\{\infty\}$ be a continuous function, such that, $f(D) \subset \mathbb{C}$ and $f_{\mid D}: D \rightarrow C$ is a holomorphic function. Then, there exists a sequence of polynomials $f_{n}(z)=\sum_{j=0}^{N_{n}} a_{j}^{n} z^{j}, a_{j}^{n} \in \mathbb{C}, N_{n} \in\{0,1,2, \ldots\}$ so that $f_{n} \rightarrow f$ uniformly on $\bar{D}$ with respect to the metric $\chi$.

Proof. Let $\varepsilon>0$. Continuity of $f$ on the compact set $\bar{D}$ implies uniform continuity with respect to the metric $\chi$. Thus, there exists $r, 0<r<1$, so that $\chi(f(z), f(r z))<$ $\frac{\varepsilon}{2}$ for all $z \in \bar{D}$. We consider the Taylor development of $f$ in $D$ with center zero $f(z)=\sum_{j=0}^{\infty} a_{j} z^{j}$. The convergence is uniform on $\{z:|z| \leq r\}$ with respect to the usual Eucledian metric in $\mathbb{C}=\mathbb{R}^{2}$. Thus, there exists $N$ so that

$$
\left|f(\tau)-\sum_{j=0}^{N} a_{j} \tau^{j}\right|<\frac{\varepsilon}{2}, \text { for all } \tau \text { with }|\tau| \leq r .
$$

But $\chi\left(z_{1}, z_{2}\right) \leq\left|z_{1}-z_{2}\right|$ and $|r z| \leq r$ for all $z \in \bar{D}$. It follows

$$
\chi\left(f(r z), \sum_{j=0}^{N} a_{j} r^{j} z^{j}\right)<\frac{\varepsilon}{2} \text { for all } z \in \bar{D} .
$$

The triangular inequality implies $\chi(f(z), P(z))<\varepsilon$ for all $z \in \bar{D}$ where $P$ is the polynomial $P(z)=\sum_{j=0}^{N} a_{j} r^{j} z^{j}$. This completes the proof.

Definition 2.2. $\widetilde{A}(D)$ denotes the set of all functions $f: \bar{D} \rightarrow \mathbb{C} \cup\{\infty\}$ such that $f$ is identically equal to $\infty$ of $f$ is continuous with respect to the metric $\chi, f(D) \subset \mathbb{C}$ and $f_{\mid D}: D \rightarrow \mathbb{C}$ is holomorphic in $D$.

The class $\widetilde{A}(D)$ contains the set of polynomials and we saw previously that polynomials are dense in $\widetilde{A}(D)$, for the topology of uniform convergence on $\bar{D}$ with respect to the metric $\chi$.

Continuing our effort to identify all uniform limits on $\bar{D}$ with respect to the metric $\chi$ by functions of the form $P(z)=\sum_{j=0}^{N} a_{j} \frac{1}{(z-w)^{j}}$, where $w \in \mathbb{C}$ is fixed, we can say the following. 
If $|w|>1$ then the set of limits is $\widetilde{A}(D)$, the same as for $w=\infty$. The only modification is in the proof of Theorem 2.1. Instead of approximating $f$ on $\{z:|z| \leq r\}$ by a partial sum of the Taylor development, we can use Runge's Theorem and make approximation by a polynomial in $\frac{1}{z-r w}$. If $|w|<1$, then the set of possible limits is the set of $f \equiv \infty$ or $f(z)=Q\left(\frac{1}{z-w}\right)$, where $Q$ is any polynomial; that is, the set of limits is almost the same as the set of approximating functions. To prove this, assume $f \not \equiv \infty$. Then, Hurwitz Theorem implies that $f(D-\{w\}) \subset \mathbb{C}$. We already used this fact which states that the limiting function $f$ can not take the value $\infty$ at an interior point where the approximating functions take finite values, unless $f \equiv \infty$. Since $f$ is holomorphic in $D-\{w\}$, then there exist unic functions $f_{1}$ and $f_{2}$ so that $f=f_{1}+f_{2}$ on $D-\{w\}, f_{1}$ is holomorphic in $D$ and $f_{2}$ is holomorphic in $\mathbb{C} \backslash\{w\}$ and satisfies $\lim _{z \rightarrow \infty} f_{2}(z)=0$. This follows using the Laurent decomposition of $f$ with center $w$; see also [4]. If $0<r_{1}<r_{2}$ are such that $\left\{\zeta:|\zeta-w| \leq r_{2}\right\} \subset D$, then $f_{1}(z)=\frac{1}{2 \pi i} \int_{|\zeta-w|=r_{2}} \frac{f(\zeta)}{\zeta-z} d \zeta$ for $|z-w| \leq r_{1}$.

Suppose $P_{n}\left(\frac{1}{z-w}\right) \rightarrow f(z)$ uniformly on $\bar{D}$ with respect to $\chi$, where $P_{n}$ are polynomials. Since $f$ takes finite values on $\left\{\zeta:|\zeta-w|=r_{2}\right\}$ whose set is a compact set not containing $\infty$, it follows easily that $P_{n}\left(\frac{1}{\zeta-w}\right) \rightarrow f(\zeta)$ uniformly on $\{\zeta:|\zeta-w|=$ $\left.r_{2}\right\}$ with respect to the usual Euclidean metric on $\mathbb{C}=\mathbb{R}^{2}$. It follows that

$$
\frac{1}{2 \pi i} \int_{|\zeta-w|=r_{2}} \frac{P_{n}\left(\frac{1}{\zeta-w}\right)}{\zeta-z} d \zeta \rightarrow f_{1}(z) \text { on }\left\{z:|z-w| \leq r_{1}\right\} .
$$

But the left hand side coincide with the constant term of the polynomial $P_{n}$. So $f_{1}$ is constant on $\left\{z:|z-w| \leq r_{1}\right\}$ and on $\bar{D}$ by analytic continuation and continuity. Thus, $f_{1}(z) \equiv c \in \mathbb{C}$. The function $f_{2}$ coincides with the principal part of the Laurent development of $f$ with center $w$. $w$ is a pole or a removable singularity for $f$. If $w$ is a pole for $f$, we conclude $f_{2}(z)=\sum_{j=1}^{N} b_{j} \frac{1}{(z-w)^{3}}$ with $N=\{1,2, \ldots\}, b_{j} \in \mathbb{C}$. If $w$ is a removable singularity for $f$ we conclude $f_{2} \equiv 0$. This completes our investigation in the case $|w|<1$. It remains the case $|w|=1$. In this case the functions $f \equiv \infty$ or $f(z)=P\left(\frac{1}{z-w}\right)$ with $P$ any polynomial are among the limiting functions. If $f$ is a limiting function $f \not \equiv \infty$, then $f(D) \subset \mathbb{C}$ and the value $\infty$ is possible only on the unit circle $T$.

Question: Is there any limiting function $f$ in the case $|w|=1$, such that $f \not \equiv \infty$ and $f(\zeta)=\infty$ for some $\zeta:|\zeta|=1, \zeta \neq w$ ? What is a characterization of the set of limiting functions in this case? 


\section{Properties of the members of $\widetilde{A}(D)$}

Proposition 3.1. Let $f \in \widetilde{A}(D)$ and $c \in \mathbb{C} \cup\{\infty\}$. If $f(\zeta)=c$ holds for a set of positive Lebesgue measure on the unit circle $T$, then $f \equiv c$.

Proof. If $c \in \mathbb{C}$, then the result follows by Privalov's Theorem ([5] page 84) applied to the function $f-c$. Let $c=\infty$. Then, by uniform continuity of $f$ with respect to the metric $\chi$, there exists $\theta_{1}<\theta_{2}<\theta_{1}+2 \pi$ and $r, 0<r<1$, so that $f(z) \neq 0$ on $\left\{t e^{i \vartheta}: r \leq t \leq 1, \theta_{1} \leq \vartheta \leq \theta_{2}\right\} \equiv L$ and $f(\zeta)=\infty$ on a compact subset $E$ of $\left\{e^{i \vartheta}: \theta_{1} \leq \vartheta \leq \theta_{2}\right\}$ with positive length. Assume $f \not \equiv \infty$ to arrive at a contradiction.

There exists a Riemann mapping $F: D \rightarrow L^{0}$ with $F^{\prime} \in H^{1}$, because $\partial L$ is rectifiable ([6] page 44). For the length of $E,|E|>0$, we have $|E|=\int_{F^{-1}(E)} F^{\prime}\left(e^{i \vartheta}\right) d \vartheta$. Thus, $F^{-1}(E)$ is a compact subset of $T=\partial D$ with positive length and the function $\frac{1}{f} \circ F$ vanishes on $F^{-1}(E)$. Since $\frac{1}{f} \circ F$ is holomorphic in $D$, Privalov Theorem implies $\frac{1}{f} \circ F \equiv 0$ on $\bar{D}$, which gives $f \equiv \infty$.

Proposition 3.2. Let $E \subset T=\partial D$ be a compact set with zero length. Then, there exists $f \in \widetilde{A}(D)$ so that $f^{-1}(\infty)=E$.

Proof. It is well known ([7] page 81) that there exists $g \in A(D)$, such that, $g_{\left.\right|_{E}} \equiv 1$ and $|g(z)|<1$ for all $z \in \bar{D}-E$. It suffices to set $f=\frac{1}{g-1}$.

If a compact set $E \subset T=\partial D$ has positive length, then it is not a compact of interpolation for $\widetilde{A}(D)$. Let $\zeta_{0} \in E$. We consider the function $h\left(\zeta_{0}\right)=1, h(\zeta)=0$ for $\zeta \in E \backslash\left\{e^{i \vartheta} \zeta_{0}:|\vartheta| \leq \varepsilon\right\}$, where $\varepsilon=\frac{|E|}{100}$, extended linearly on $\left\{e^{i \vartheta} \zeta_{0}:|\vartheta| \leq \varepsilon\right\} \cap E$. This function $h$ can not have an extension $f$ in $\widetilde{A}(D)$. Since $\left|E \backslash\left\{e^{i \theta} \zeta_{0}:|\vartheta| \leq \varepsilon\right\}\right|>0$, it would follow that $f \equiv 0$; but $f\left(\zeta_{0}\right)=h\left(\zeta_{0}\right)=1 \neq 0$. This gives a contradiction.

Question: Is it true that every compact set $E \subset T=\vartheta D$ with zero length is a compact of interpolation for $\widetilde{A}(D)$ ? That is, is it true, that, for every continuous function $h: E \rightarrow \mathbb{C} \cup\{\infty\}$ there exists $f \in \widetilde{A}(D)$ so that $f_{\mid E}=h$ ? Furthermore one could ask for a characterization of compact sets $E \subset \bar{D}$ which are of interpolation for $\widetilde{A}(D)$.

We notice that for $f, g \in \widetilde{A}(D)$ we do not have in general $\sup _{z \in \bar{D}} \chi(f(z), g(z)) \leq$ $\sup \chi(f(z), g(z)) \cdot C$ for any constant $C<+\infty$ independent of $f$ and $g$. In fact, even $|z|=1$ 
if we fix $r, 0<r<1$, there is no constant $C<+\infty$ so that $\sup _{z \in \bar{D}} \chi(f(z), g(z)) \leq$ $C \sup _{r \leq|z| \leq 1} \chi(f(z), g(z))$ for all $f, g \in \widetilde{A}(D)$. To see this we set $g \equiv \infty$ and $f_{n}(z)=n z$.

Then,

$$
\begin{gathered}
\sup _{z \in \bar{D}} \chi\left(f_{n}(z), g(z)\right) \geq \chi(0, \infty)=1 \text { but } \\
\sup _{r \leq|z| \leq 1} \chi\left(f_{n}(z), g(z)\right)=\chi(n r, \infty) \rightarrow 0, \text { as } n \rightarrow+\infty .
\end{gathered}
$$

However, we have the following.

Proposition 3.3. Let $f, g \in \widetilde{A}(D)$. If $f(\zeta)=g(\zeta)$ for all $\zeta \in T=\partial D$, then $f \equiv g$.

Proof. If $f \equiv \infty$ or $g \equiv \infty$, then the result follows from Proposition 3.1. We assume $f \not \equiv \infty$ and $g \not \equiv \infty$. Proposition 3.1. implies that the set $E=\{\zeta \in T: f(\zeta)=\infty\}$ is a compact set with zero length. Since $f(\zeta)=g(\zeta)$ for all $\zeta \in T$ we conclude that the function $f-g$, which takes finite values in $D$ and is holomorphic in $D$, extends continuously on $D \cup(T \backslash E)$ with values in $\mathbb{C}$. We also have $f(z)-g(z)=0$ on $T \backslash E$. Since $T \backslash E$ contains a compact set with positive length, it follows by Privalov's Theorem ([5], page 84) that $f(z) \equiv g(z)$ on $D$ and by continuity on $\bar{D}$.

The proof is complete.

Furthermore the mean value property $f(0)=\frac{1}{2 \pi} \int_{0}^{2 \pi} f\left(e^{i \vartheta} d \vartheta\right.$ does not hold for all $f \in \widetilde{A}(D), f \not \equiv \infty$. If we set $\frac{1}{z-1}=f(z)$. Then $f \in \widetilde{A}(D)$ with $f^{-1}(\infty)=\{1\}$ and $f \notin L^{1}(T, d \vartheta)$. Thus, the mean value property is not valid. Even if we interprete the integral as a principal value we have:

$$
\lim _{\varepsilon \rightarrow 0^{+}} \frac{1}{2 \pi} \int_{\varepsilon}^{2 \pi-\varepsilon} \frac{1}{e^{i \vartheta}-1} d \vartheta=\lim _{\varepsilon \rightarrow 0^{+}} \frac{1}{2 \pi} \int_{\varepsilon}^{\pi} 2 R e \frac{1}{e^{i \theta}-1} d \vartheta=-\frac{1}{2} \neq-1=f(0),
$$

because $R e \frac{1}{e^{i \vartheta}-1}=-\frac{1}{2}$ for all $\vartheta \in \mathbb{R}$.

The mean value property $f(0)=\frac{1}{\pi} \int_{x^{2}+y^{2}<1} f(x+i y) d x d y$ is neither valid for all $f \in \widetilde{A}(D), f \not \equiv \infty$. If we set $f(z)=\frac{1}{(z-1)^{2}}$ then $f \in \widetilde{A}(D), f^{-1}(\infty)=\{1\}$ and $f \notin$ $L^{1}(D, d x d y)$. However, in polar coordinates, the iterated integral $\frac{1}{\pi} \int_{r=0}^{1} \int_{\theta=0}^{2 \pi} f\left(r e^{i \vartheta}\right)(d \vartheta)$. $r d r$ is equal to $f(0)$ for all holomorphic functions in $D$. I also think that for every $G \in H_{0}^{1}$ the formula $f(0)=\frac{1}{2 \pi} \int_{\vartheta=0}^{2 \pi} f\left(e^{i \vartheta}\right)\left(1+G\left(e^{i \vartheta}\right)\right) d \vartheta$ does not hold for all $f \in \widetilde{A}(D)$, $f \not \equiv 0$, but I do not have a proof. 


\section{Topological properties of $\widetilde{A}(D)$}

In the disc algebra $A(D)=\{f: \bar{D} \rightarrow \mathbb{C}$ continuous on $\bar{D}$ and holomorphic in $D\}$ we consider the metric $d(f, g)=\max _{|z| \leq 1}|f(z)-g(z)|$. Then $(A(D), d)$ is a complete metric space. In $\widetilde{A}(D)$ we consider the metric $\widetilde{d}(f, g)=\max _{|z| \leq 1} \chi(f(z), g(z))$. Very often, we write $\chi(f, g)$ instead of $\widetilde{d}(f, g), f, g \in \widetilde{A}(D)$. Then $(A(D), \widetilde{d})$ is a complete metric space. If $f_{n} \in \widetilde{A}(D)$ is $\widetilde{d}$-Cauchy sequence, then, since $(\mathbb{C} \cup\{\infty\}, \chi)$ is a complete metric space, there exists a function $f: \bar{D} \rightarrow \mathbb{C} \cup\{\infty\}$, such that, for every $z \in \bar{D}$ $\chi\left(f_{n}(z), f(z)\right) \rightarrow 0$, as $n \rightarrow+\infty$. Let $\varepsilon>0$, then $\sup \chi\left(f_{n}(z), f_{m}(z)\right)<\varepsilon$ for all $n, m \geq n_{0}$, for some $n_{0}$. Making $m \rightarrow+\infty$ we obtain $\widetilde{d}\left(f_{n}, f\right)<\varepsilon$ for all $n \geq n_{0}$. So $\widetilde{d}\left(f_{n}, f\right) \rightarrow 0$. If $f \equiv \infty$, then $f \in \widetilde{A}(D)$.

Assume $f\left(z_{0}\right) \in \mathbb{C}$ for some $z_{0} \in \bar{D}$.

Thus $f_{n}\left(z_{0}\right) \in \mathbb{C}$ for all $n \geq n_{1}$ for some $n_{1}$. On a compact neighborhood $V_{z_{0}}$ of $z_{0}$ in $\bar{D}$ we find $|f(z)| \leq M$ for some $M<+\infty$ and for all $z \in \widetilde{V}_{z_{0}}$ where $\widetilde{V}_{z_{0}}$ is a compact neighborhood of $z_{0}$ included in $V_{z_{0}}$.

We set $\varepsilon=\frac{1}{2} \chi(\infty,\{a \in \mathbb{C}:|\alpha| \leq M\})>0$. Then, the set $\{\beta \in \mathbb{C} \cup\{\infty\}: \chi(\beta,\{a \in$ $\mathbb{C}:|\alpha| \leq M\}) \leq \varepsilon\}$ is compact and does not contain $\infty$. But every compact subset of $\mathbb{C}$ is bounded (in Eucledian distance). So there exists $M^{\prime}, M \leq M^{\prime}<+\infty$, so that, $\chi(\beta, \alpha) \leq \varepsilon$ for some $\alpha$ with $|\alpha| \leq M$ implies $|\beta| \leq M^{\prime}$. Thus, there exist $n_{1}$ so that $\left|f_{n}(z)\right| \leq M^{\prime}$ and $|f(z)| \leq M^{\prime}$ for all $z \in \widetilde{V}_{z_{0}}$ and $n \geq n_{1}$. Thus $\chi\left(f_{n}(z), f(z)\right) \geq$ $\frac{\left|f_{n}(z)-f(z)\right|}{1+M^{\prime 2}}$. So $f_{n} \rightarrow f$ uniformly with respect to Eucledian distance on $\widetilde{V}_{z_{0}}$. It follows that $f$ is holomorphic in some open set $U_{z_{0}}$ containing $z_{0}$, if $z_{0} \in D$ or $z_{0} \in \partial U_{z_{0}}$ if $z_{0} \in T$.

Assume $z_{1} \in D$ is such that $f\left(z_{1}\right)=\infty$. Then on a compact neighborhood $U_{z_{1}}$ of $z_{1}$ we have $\chi(f(z), \infty)<\frac{1}{3}$. There exists $n_{3}$ so that $\chi\left(f(z), f_{n}(z)\right)<\frac{1}{3}$ for all $z \in U_{z}$, and $n \geq n_{3}$. The triangular inequality implies $\chi\left(f_{n}(z), \infty\right)<\frac{2}{3}$ for all $z \in U_{z}$, and $n \geq n_{3}$. This implies $\left|f_{n}(z)\right|>\sqrt{\frac{7}{2}}$ and $|f(z)|>\sqrt{\frac{7}{2}}$. Thus, in the interior of $U_{z_{1}}$ the holomorphic functions $\frac{1}{f_{n}}$ and $\frac{1}{f}$ are bounded above by $\sqrt{\frac{2}{7}}$. Since $\chi\left(\frac{1}{\alpha}, \frac{1}{\beta}\right)=\chi(\alpha, \beta)$ we see $\frac{1}{f_{n}} \rightarrow \frac{1}{f}$ uniformly on $U_{z_{1}}$ with respect to the metric $\chi$; but $\chi\left(\frac{1}{f_{n}(2)}, \frac{1}{f(z)}\right) \geq$ $\frac{\left|\frac{1}{f_{n}(z)}-\frac{1}{f(z)}\right|}{\frac{9}{7}}$ on $U_{z_{1}}$. This implies that $\frac{1}{f_{n}} \rightarrow \frac{1}{f}$ uniformly on $U_{z_{1}}$ with respect to the usual Eucledian metric on $\mathbb{C}=\mathbb{R}^{2}$. If $f_{n} \equiv \infty$ for infinitely many $n$ 's, then obviously $f \equiv \infty$ belongs to $\widetilde{A}(D)$. So we assume $f_{n}(D) \subset \mathbb{C}$ and $f_{n \mid D}$ holomorphic for every 
n. But $\frac{1}{f_{n}(z)} \neq 0$ for every $n$ and $z \in U_{z_{1}}$ while $\frac{1}{f\left(z_{1}\right)}=0$. Hurwitz Theorem implies $f(z) \equiv \infty$ on $U_{z_{1}}$. Thus the set $\{z \in D: f(z)=\infty\}$ is open. By the continuity of $f$, the same set is closed in $D$. Since $D$ is connected, this set is either empty or the hole $D$. In the second case $f \equiv \infty$, so $f \in \widetilde{A}(D)$. In the first case $f(D) \subset \mathbb{C}$ and $f$ is holomorphic in $D$. Since $f: \bar{D} \rightarrow \mathbb{C} \cup\{\infty\}$ is continuous we conclude that $f \in \widetilde{A}(D)$. This completes the proof.

Thus, we have proved the following.

Theorem 4.1. $(\widetilde{A}(D), \widetilde{d})$ is a complete metric space.

Proposition 4.2. $A(D)$ is an open dense subset of $\widetilde{A}(D)$. Furthermore, the relative topology of $A(D)$ from $\widetilde{A}(D)$ coincides with the usual topology of $A(D)$.

Proof. Let $f \in A(D)$; then $f(\bar{D})$ is a compact subset of $\mathbb{C}$ and does not contain $\infty$.

We set $\varepsilon=\frac{1}{2} \chi(f(\bar{D}), \infty)>0$. If $g \in \widetilde{A}(D)$ satisfies $\sup \chi(f(z), g(z))<\varepsilon$, then it follows that $\infty \notin g(\bar{D})$. So $g \in A(D)$. This proves that $A(D)$ is open in $\widetilde{A}(D)$. Since $A(D)$ contain the polynomials Theorem 2.1. implies that $A(D)$ is dense in $\widetilde{A}(D)$.

Let $f_{n} \in A(D)$ and $f \in A(D)$ be such that $f_{n} \rightarrow f$ uniformly on $\bar{D}$ with respect to the Eucledian metric in $\mathbb{C}=\mathbb{R}^{2}$. Since $\chi(\alpha, \beta) \leq|\alpha-\beta|$, it follows that $f_{n} \rightarrow f$ in $\widetilde{A}(D)$.

Reversely, let $g_{n} \in A(D)$ and $g \in A(D)$ be such that $g_{n} \rightarrow g$ in $\widetilde{A}(D)$. Since $g(\bar{D})$ is a compact subset of $\mathbb{C}$, we find $|g(z)| \leq M$ for all $z \in \bar{D}$ and some $M<+\infty$. By an argument already used several times we have $\left|g_{n}(z)\right| \leq M^{\prime}$ for all $z \in \bar{D}$ and $n \geq n_{5}$ for some $n_{5}$ and $M^{\prime}, M \leq M^{\prime}<+\infty$. Thus, the convergence $g_{n} \rightarrow g$ in $\widetilde{A}$ implies the convergence $g_{n} \rightarrow g$ in $A(D)$. This proves that the relative topology of $A(D)$ from $\widetilde{A}(D)$ coincides with the usual topology of $A(D)$.

In [8] we consider any Hausdorff measure function $h$. We follow the notations of [8]. Let $f \in \widetilde{A}(D)$. Then $E_{f}=\{\zeta \in T: f(\zeta) \notin f(D)\}$. It is proven ([8]) that the set of $f \in A(D)$ such that $\Lambda_{h}\left(E_{f}\right)=0$ is dense and $G_{\delta}$ in $A(D)$. By Proposition 4.2. the same set is dense and $G_{\delta}$ in $\widetilde{A}(D)$. Thus, the set of $f \in \widetilde{A}(D)$ such that $\Lambda_{h}\left(E_{f}\right)=0$ is residual in $\widetilde{A}(D)$.

Proposition 4.3. Let $h$ be any Hausdorff measure function. The set of all $f \in \widetilde{A}(D)$, such that $\Lambda_{h}\left(E_{f}\right)=0$ is dense and $G_{\delta}$ in $\widetilde{A}(D)$, where $E_{f}=\{\zeta \in T: f(\zeta) \notin f(D)\}$. 
Proof. After the previous discussion, it remains to prove that this set is $G_{d}$ in $\widetilde{A}(D)$. We follow the notation of [8], especially of the first part of the proof of Theorem 3.1 of [8]. We consider the sets $S_{N}=\left\{f \in \widetilde{A}(D): M_{h}\left(E_{f}\right)<\frac{1}{N}\right\}$.

Since $\bigcap_{N=1}^{\infty} S_{N}$ is our set, it suffices to show that $S_{N}-\{f \equiv \infty\}$ is open. Since the singleton $\{f \equiv \infty\}$ is a $G_{\delta}$, the result would follow, because the union of two $G_{\delta}$ sets is again a $G_{\delta}$ set.

Let $f \in S_{N}(f \not \equiv \infty)$; thus, there exists a countable collection of discs $D_{m}$ so that $E_{f} \subset \bigcup_{m} D_{m}$ and $\Sigma h\left(r_{m}\right)<\frac{1}{N}$ where $r_{m}$ is the radius of $D_{m}$. We have $f \not \equiv \infty$.

Since $E_{f} \supset f^{-1}(\infty)$, by the continuity of $f$ and the compactness of $T \backslash \bigcup_{m} D_{m}$, there exists $M<+\infty$ so that $|f(\zeta)|<M \forall \zeta \in T \backslash \cup D_{m}$.

We consider a finite set of disjoint open intervals $\left(\alpha_{i}, \beta_{i}\right) i=1, \ldots, n, \alpha_{1}<\beta_{1}<$ $\alpha_{2}<\beta_{2}<\cdots<\alpha_{n}<\beta_{n}<\alpha_{1}+2 \pi$ and an $r, 0<r<1$ so that $f^{-1}(\infty) \subset \bigcup_{i=1}^{n}\left\{e^{i \vartheta}\right.$ : $\left.\alpha_{i}<\vartheta<\beta_{i}\right\} \subset \bigcup_{m} D_{m}$ and, for every $z \in S=\left\{t e^{i \theta}: r \leq t \leq 1, \theta \in \bigcup_{i=1}^{n}\left(\alpha_{i}, \beta_{i}\right)\right\}$ we have $|f(z)|>\widetilde{M}$ for some $\widetilde{M}, M<\widetilde{M}<+\infty$.

Obviously, for all $\zeta \in T \backslash \bigcup_{m} D_{m}$ we have $f(\zeta) \notin f(S)$. As $\zeta \notin E_{f}$, it follows $f(\zeta) \in f(D \backslash S)$; thus, $\delta_{f}(f(\zeta), D \backslash S)>0$. By continuity of $\delta_{f}$ and compactness of $T \backslash \bigcup_{m} D_{m}$ we have that $\eta=\inf \left\{\delta_{f}(f(\zeta), D \backslash S) ; \zeta \in T \backslash \bigcup_{m} D_{m}\right\}>0$. As in [8], if $g \in \widetilde{A}(D)$ satisfies $\sup _{D \backslash S}|f(z)-g(z)|<\frac{\eta}{2}$ then, it follows $\delta_{g}(g(\zeta) ; D \backslash S)>0$ for all $\zeta \in T \backslash \bigcup_{m} D_{m}$. Thus $g(\zeta) \in g(D \backslash S) \subset g(D)$ and $E_{g} \subset \bigcup_{m} D_{m}$, which would imply $g \in S_{N}$.

So it suffices to find $\varepsilon>0$, so that $\sup _{|z| \leq 1} \chi(f(z), g(z))<\varepsilon$ implies $\sup _{z \in D \backslash S} \mid f(z)-$ $g(z) \mid<\frac{\eta}{2}$. Since $f(\overline{D \backslash S}) \subset \mathbb{C}$ we find $M_{1}<+\infty$ so that $|f(z)|<M_{1}$ for all $z \in \overline{D \backslash S}$. There exists $\varepsilon_{1}>0$ so that $|a|<M_{1}, \chi(\alpha, \beta)<\varepsilon_{1}$ imply $|\beta|<M_{1}+1=M_{2}$. So, if $\chi(f, g)<\varepsilon_{1}, g \in \widetilde{A}(D)$ we have $|g(z)|,|f(z)| \leq M_{2}$ for all $z \in D \backslash S$. Now for $z \in D \backslash S$ we have

$$
\chi(f(z), g(z))=\frac{|f(z)-g(z)|}{\sqrt{1+|f(z)|^{2}} \sqrt{1+|g(z)|^{2}}}>\frac{|f(z)-g(z)|}{1+M_{2}^{2}} .
$$

We set $\varepsilon=\frac{1}{2} \min \left(\varepsilon_{1}, \frac{\eta}{2\left(1+M_{2}^{2}\right)}, \chi(f, \infty)\right)>0$. We can easily verify that, if $g \in \widetilde{A}(D)$ is such that $\chi(f, g)<\varepsilon$, then, $g \not \equiv \infty$ and for every $z \in D \backslash S$ we have $|f(z)-g(z)|<\frac{\eta}{2}$, which imply $g \in S_{N}, g \not \equiv \infty$. This proves that $S_{N}-\{f \equiv \infty\}$ is open in $\widetilde{A}(D)$. The result easily follows since the singleton $\{f \equiv \infty\}$ is a $G_{\delta}$. 
Next we consider the sets

$$
X=\{f \in A(D): f(D) \subset f(T)\} \subset A(D)
$$

and

$$
Y=\{f \in \widetilde{A}(D): f(D) \subset f(T)\} \subset \widetilde{A}(D) .
$$

G. Costakis pointed out that $X$ is closed in $A(D)$. In fact $Y$ is also closed in $\widetilde{A}(D)$. It suffices to show that $\widetilde{A}(D) \backslash Y$ is open in $\widetilde{A}(D)$.

Proposition 4.4. $Y$ is closed in $\widetilde{A}(D)$.

Proof. Let $f \in \widetilde{A}(D) \backslash Y$; then, there exists $z_{0} \in D$ so that $f\left(z_{0}\right) \in \mathbb{C}$ and $f\left(z_{0}\right) \notin f(T)$. Since $f(T)$ is a compact subset of $\mathbb{C} \cup\{\infty\}$, we find $\varepsilon>0$ so that $\chi\left(f\left(z_{0}\right), f(T)\right)>\varepsilon$. Let $r>0$ so that $\left\{z:\left|z_{0}-z\right| \leq r\right\} \subset D$ and $\chi(f(z), f(z))<\varepsilon$ for all $z:\left|z_{0}-z\right| \leq r$. Since $f$ is non-constant we may choose $r^{\prime}: 0<r^{\prime} \leq r$ so that $f(z) \neq f\left(z_{0}\right)$ for all $z: 0<$ $\left|z-z_{0}\right| \leq r^{\prime}$. Let $\delta=\min _{\left|z-z_{0}\right|=r^{\prime}}\left|f(z)-f\left(z_{0}\right)\right|>0$. Since $\left\{f(z):\left|z-z_{0}\right|=r^{\prime}\right\}$ is a compact subset of $\mathbb{C}$, we find $\varepsilon^{\prime}, 0<\varepsilon^{\prime}<\varepsilon$, so that $\chi(f(z), w)<\varepsilon^{\prime}$ for some $z:\left|z-z_{0}\right|=r$ implies $|f(z)-w|<\delta$. We will show that if $g \in A(D)$ and $\sup _{z \in \bar{D}} \chi(f(z), g(z))<\varepsilon^{\prime}$, then $g \in \widetilde{A}(D) \backslash Y$.

It suffices to show that $f\left(z_{0}\right) \in g(D) \backslash g(T)$. Since, for every $e^{i \vartheta} \in T$ we have $\chi\left(g\left(e^{i \vartheta}\right), f\left(e^{i \vartheta}\right)\right)<\varepsilon^{\prime}$; so $\chi\left(g\left(e^{i \vartheta}\right), f(T)\right)<\varepsilon^{\prime}$; but $\chi\left(f\left(z_{0}\right), f(T)\right)>\varepsilon>\varepsilon^{\prime}$, so $g\left(e^{i \vartheta}\right) \neq$ $f\left(z_{0}\right)$ for all $\vartheta \in \mathbb{R}$. It follows $f\left(z_{0}\right) \notin g(T)$.

We also have

$$
\left|f(z)-f\left(z_{0}\right)\right| \geq \delta>|f(z)-g(z)| \text { for all } z:\left|z-z_{0}\right|=r^{\prime}
$$

Rouche's lemma implies that $f\left(z_{0}\right) \in g(D)$. This completes the proof.

$\widetilde{A}(D) \backslash Y$ contains every non-constant polynomial. Now every constant $a \in \mathbb{C}$ is the limit in $\widetilde{A}(D)$ of the sequence $P_{n}=a+\frac{z}{n}$. Theorem 2.1. implies the $\widetilde{A}(D) \backslash Y$ is dense; as, it is open also, it follows that $Y$ is a closed subset of $\widetilde{A}(D)$ which is of the first category. In a similar may one can prove that $X$ is a closed subset of $A(D)$ which is of the first category.

We notice that $X \subset Y$ are non-void. To see this, we consider $K \subset T$ a compact set of Cantor type with zero length. It is well known that there exists a continuous mapping $\Phi: K \rightarrow[0,1]$ with $\Phi(K)=[0,1]$. Next we consider a Peano curve $\Gamma:[0,1] \rightarrow \bar{D}$ which is continuous and $\Gamma([0,1])=\bar{D}$. 
We consider the function $h=\Gamma \circ \Phi: K \rightarrow \bar{D}$, which is continuous and $h(K)=\bar{D}$. By the Rudin-Carleson Theorem ([7], pages 81-82) there exists $f \in A(D)$ so that $f_{\mid K}=h$ and $|f(z)| \leq 1$ for all $x \in \bar{D}$. Then $f(D) \subset \bar{D}=h(K)=f(K) \subset f(T)$. Thus $f \in X \subset Y$ and $X, Y$ are non-void. Summarizing we have proved

Proposition 4.5. $Y$ is a non-void closed subset of $\widetilde{A}(D)$ of first category.

Furthermore, we consider the set $W \subset Y, W=\{f \in \widetilde{A}(D): f(T)=\mathbb{C} \cup\{\infty\}\} \subset Y$. Obviously, $W$ is of first category in $\widetilde{A}(D)$. In fact $W$ is closed in $\widetilde{A}(D)$. It suffices to show that $\widetilde{A}(D) \backslash W$ is open. Let $f \in \widetilde{A}(D) \backslash W$. Then, there exist $P \in \mathbb{C} \cup\{\infty\}$ such that $P \notin f(T)$. Since $f(T)$ is compact $\chi(P, f(T))=\delta>0$. Let $0<\varepsilon<\delta$. Then, if $g \in \widetilde{A}(D)$ satisfies $\sup _{\bar{D}} \chi(f(z), g(z))<\varepsilon$, it follows easily that $P \notin g(T)$. Thus, $g \in \widetilde{A}(D) \backslash W$ and $\widetilde{A}(D) \backslash W$ is open in $\widetilde{A}(D)$. It follows that $W$ is a closed set of first category in $\widetilde{A}(D)$. We have proved

Proposition 4.6. $W$ is a closed subset of $A(D)$ of first category.

We do not know if $W \neq \emptyset$. If it is true that every compact set $K \subset T$ with zero length, is a compact of interpolation for $\widetilde{A}(D)$, then we can show that $W \neq \emptyset$.

We consider $K_{1} \subset T$ a compact set of Cantor type with length $0 . K_{2} \subset T$ is disjoint from $K_{1}$, it is a compact set of Cantor type with length 0 , which is contained in the middle third in the first level of the construction of $K_{1} . K_{2}$ has as middle point the middle point $P$ of $K . K_{n+1} \subset T$ is a compact set of Cantor type with zero length, disjoint from all $K_{1}, \ldots, K_{n}$, lying in the middle third in the first level of construction of $K_{n}$ and with middle point $P$.

We set $K=\{P\} \cup \bigcup_{i=1}^{\infty} K_{i}$. This set is compact with zero length. So, we assume that it is of interpolation for $\widetilde{A}(D)$. There exist continuous maps $\Phi_{i}: K_{i} \rightarrow[2 i-1,2 i]$ so that $\Phi_{i}\left(K_{i}\right)=[2 i-1,2 i]$. There exist Peano curves $\Gamma_{i}:[2 i-1,2 i] \rightarrow\{z: i-1 \leq|z| \leq i\}$, $i=1,2, \ldots$ such that $\Gamma_{i}([2 i-1,2 i])=\{z: i-1 \leq|z| \leq i\}$. We consider the maps $\Gamma_{i} \circ \Phi_{i}: K_{i} \rightarrow\{z: i-1 \leq|z| \leq i\}$ which are continuous and onto. The map $h: K \rightarrow \mathbb{C} \cup\{\infty\}$ defined by $h(P)=\infty$ and $h_{\mid K_{i}}=\Gamma_{i} \circ \Phi_{i}$ is continuous and onto; thus $h(K)=\mathbb{C} \cup\{\infty\}$. By assumption there exist $f \in \widetilde{A}(D)$ so that $f_{\mid K}=h$.

Then $f \in W$ because $f(T) \supset f(K)=h(K)=\mathbb{C}-\{\infty\}$. So $W$ is non void. 


\section{Further results and open questions}

One open question is if Mergelyan's Theorem extends to our case. That is, to investigate the set of uniform limits with respect to the distance $\chi$ of polynomials on a compact set $L \subset \mathbb{C}$ with $L^{c}$ connected.

The question is if the limits are exactly the continuous functions $f: L \rightarrow \mathbb{C} \cup\{\infty\}$ such that, for every compoment $V$ of $L^{0}$ either $f_{\mid V} \equiv \infty$ or $f(V) \subset \mathbb{C}$ and $f_{\mid V}$ is holomorphic. We notice that, it is possible so that for one component we have the first alternative, while for another component we may have the second alternative. The general case is open. We just give an affirmative answer in a few particular cases.

Proposition 5.1. Let $L \subset \mathbb{C}$ be a compact set, such that, $L=\overline{L^{0}}$ and $L^{0}$ is starlike with respect to some point $z_{0} \in L^{0}$. Then the uniform limits with respect to the metric $\chi$ of polynomials on $L$ are exactly the functions $f \equiv \infty$ of $f: L \rightarrow \mathbb{C} \cup\{\infty\}$ continuous with $f\left(L^{0}\right) \subset \mathbb{C}$ and $f_{\mid L^{0}}$ holomorphic.

For the proof we may assume $z_{0}=0$. Then we imitate the proof on $L=\bar{D}$. The difference is that when we approximate $f$ on the compact set $r L$, with $0<r<1$, we cannot use a Taylor development of $f$, but we can use the classical Mergelyan's Theorem. The approximation is uniform on $r L$ with respect to the Eucledian distance on $\mathbb{C}=\mathbb{R}^{2}$; since $\chi(\alpha, \beta) \leq|\alpha-\beta|$ for all $\alpha, \beta \in \mathbb{C}$, this implies approximation uniform with respect to the distance $\chi$.

Under the assumptions of Proposition 5.1., if $w \in \mathbb{C} \backslash L$, then the uniform limits on $L$ by functions of the form $P\left(\frac{1}{z-w}\right)$, where $P$ is any polynomial are the same with those given by Proposition 5.1. The difference in the proof is, when we apply Mergelyan's Theorem to do approximation of $f$ on $r L, 0<r<1$, then we choose as pole $r w \notin r L$ and not $\infty$.

Next we consider the analogue problem on an annulus. Without loss of generality we may assume that this annulus is $\Omega=\mathcal{D}(0, r, 1)=\{z \in \mathbb{C}: r<|z|<1\}$ with $0<r<1$.

Proposition 5.2. Let $0<r<1$ and $\Omega=\mathcal{D}(0, r, 1)$. Then the uniform limits on $\bar{\Omega}$ with respect to $\chi$ of polynomials are the functions $f \in \widetilde{A}(D)$.

In the proof, if the sequence of polynomials $P_{n}$ converges uniformly on $\overline{\mathcal{D}(0, r, 1)}$ with respect to $\chi$ to $f \not \equiv \infty$, then $f\{z: r<|z|<1\} \subset \mathbb{C}$ and $f$ is holomorphic in 
$\{z: r<|z|<1\}$. So there exists $M<+\infty$, so that $|f(z)|<M$ on $|z|=\frac{r+1}{2}$. Because $\chi\left(P_{n}, f\right) \rightarrow 0$ we find $M_{1}: M<M_{1}<+\infty$ and $n_{0}$ so that $\mid P_{n}(z)<M_{1}$ for $|z|=\frac{1+r}{2}$ and $n \geq n_{0}$. For $|a|,|b|<M_{1}$ we have $\chi(\alpha, \beta) \geq \frac{|a l-\beta|}{1+M_{1}^{2}}$; So $P_{n} \rightarrow f$ uniformly on $|z|=\frac{1+r}{2}$ with respect to the usual Euclidian metric on $\mathbb{C}=\mathbb{R}^{2}$. Thus, the sequence $P_{n}, n=1,2, \ldots$ is uniformly Cauchy on $|z|=\frac{r+1}{2}$ with respect to the Eucledian metric in $\mathbb{C}$. The maximum principle implies that the sequence of polynomials $P_{n}$ is uniformly Cauchy on $|z| \leq \frac{r+1}{2}$. Its limit is a holomorphic extension of $f$, so finally $f \in \widetilde{A}(D)$. It is remarkable that the value $\infty$ is not permitted on $|z|=r$, unless $f \equiv \infty$.

If we wish to investigate the analogue problem on $\overline{\mathcal{D}(0, r, 1)}, 0<r<1$ using functions of the form $P\left(\frac{1}{z-w}\right)$ where $P$ is any polynomial and $w \in \mathbb{C}$ fixed, then the answer is the following.

If $|w|>1$ then the possible limits are $f \in \widetilde{A}(D)$. The proof is the same as in the case of polynomials.

The case $|w|<r$ is reduced to the previous one by an inversion. The possible limits are $f \equiv \infty$ or $f:\{z: r \leq|z|\} \rightarrow C \cup\{\infty\}$ continuous with $f(\{z: r<|z|\}) \subset \mathbb{C}$ and $f$ holomorphic in $r<|z|$ and $\lim _{z \rightarrow \infty} f(z)$ exist in $\mathbb{C}$.

In the case $r<|w|<1$, the limits are exactly $f \equiv \infty$ or $f(z)=P\left(\frac{1}{z-w}\right)$ with $P$ any polynomial. The argument in the proof uses Cauchy transforms as in $\S 2$. In the case $|w|=1$ or $|w|=r$ I do not know the answer.

For $r=1$, I do not know the answer to the analogue question of finding the uniform limits on $T=\{z \in \mathbb{C}:|z|=1\}$ with respect to $\chi$ of all polynomials.

Finally we may consider $\bar{\Omega}=\overline{\mathcal{D}(0, r, 1)} 0<r<1$ and searching for the limits using two poles. There are 5 cases $\{\infty\} \cup\{w \in \mathbb{C}:|w|>1\},\{w \in \mathbb{C}:|w|=1\}$, $\{w \in \mathbb{C}: r<w<1\},\{w \in \mathbb{C}:|w|=r\},\{w \in \mathbb{C}:|2|<r\}$. So the number of possible locations of two poles $w_{1}, w_{2}$ is $\left(\begin{array}{l}5 \\ 2\end{array}\right)=10$. In some of these cases the author does not know the answer, but in some other cases we know the answer.

If we use the poles $w_{1}=\infty, w_{2}=0$ then we approximate by functions of the form $\sum_{n=-N}^{N} a_{n} z^{n}$. The limit functions are $f \equiv \infty$ or $f: \overline{\mathcal{D}(0, r, 1)} \rightarrow \mathbb{C} \cup\{\infty\}$ continuous, $f(\mathcal{D}(0, r, 1)) \subset \mathbb{C}$ and $f_{\mid \mathcal{D}(0, r, 1)}$ holomorphic.

For the proof we use Laurent expansion and we approximate in $\chi$ metric separately the principal part and the regular part. Next we need a lemma of the following form.

Lemma 5.3. Let $g, f: \mathcal{D}(0, r, 1) \rightarrow \mathbb{C} \cup\{\infty\}$ are continuous on $\overline{\mathcal{D}(0, r, 1)}, f(\{z: r \leq$ 
$|z|<1\}) \subset \mathbb{C}, g(\{z: r<|z| \leq 1\}) \subset \mathbb{C}, f_{n}, g_{n}: \overline{\mathcal{D}(0, r, 1)} \rightarrow \mathbb{C}$ continuous.

We assume that

$$
\sup _{z \in \overline{\mathcal{D}(0, r, 1)}} \chi\left(f_{n}(z), f(z)\right) \rightarrow 0 \text { and } \sup _{z \in \overline{\mathcal{D}(0, r, 1)}} \chi\left(g_{n}(z), g(z)\right) \rightarrow 0
$$

Then $f+g$ is well defined with values in $\mathbb{C} \cup\{\infty\}$ and

$$
\sup _{z \in \overline{\mathcal{D}(0, r, 1)}} \chi\left(f_{n}(z)+g_{n}(z), f(z)+g(z)\right) \rightarrow 0
$$

For the proof of the lemma it is essential that $f^{-1}(\infty) \subset\{z:|z|=1\}$ and $g^{-1}(\infty) \subset$ $\{z:|z|=r\}$; so the compact sets $f^{-1}(\infty), g^{-1}(\infty)$ are disjoint.

We fix $\varepsilon>0$ and we are looking for $n_{0}$ so that $\sup _{z \in \frac{\mathcal{D}(0, r, 1)}{}} \chi\left(f_{n}(z)+g_{n}(z), f(z)+g(z)\right)<$ $\varepsilon$ for all $n \geq n_{0}$.

We obtain that there exist a constant $M<+\infty$ so that $|f(z)| \leq M$ for $r \leq|z| \leq \frac{r+1}{2}$ and $|g(z)| \leq M$ for $\frac{r+1}{2} \leq|z| \leq 1$. We can choose a relatively open neighborhood $V_{1}$ of $f^{-1}(\infty)$ so that $V_{1} \subset\left\{z: \frac{r+1}{2}<|z| \leq 1\right\}$ so that $|f(z)|>M_{1}$ for $z \in V_{1}$, where $M_{1}$ is in our disposal. Then $|f(z)+g(z)|>M_{1}-M$ on $V_{1}$.

We easily find $n_{1}$ so that for $n \geq n_{1}$ we have $\left|f_{n}(z)+g_{n}(z)\right|>M_{1}-M-1$ on $V_{1}$. We can choose $M_{1}$ big enough so that the $\chi$ diameter of $\{\infty\} \cup\left\{w \in \mathbb{C}:|w|>M_{1}-M-1\right\}$ is less than $\varepsilon$. So, for $n \geq n_{1}$ we have $\sup _{z \in V_{1}} \chi\left(f_{n}(z)+g_{n}(z), f(z)+g(z)\right)<\frac{\varepsilon}{3}$. In a similar way we find a relatively open neighborhood $V_{2}$ of $g^{-1}(\infty), V_{2} \subset\left\{z: r \leq|z|<\frac{r+1}{2}\right\}$ and $n_{2}$ so that for $n \geq n_{2}$ we have $\sup _{z \in V_{2}} \chi\left(f_{n}(z)+g_{n}(z), f(z)+g(z)\right)<\frac{\varepsilon}{3}$. The set $\overline{\mathcal{D}(0, r, 1)}-$ $\left(V_{1} \cup V_{2}\right)$ is compact. By continuity there exist $M_{2}<+\infty$ so that $|f(z)|,|g(z)|<M_{2}$ on $\overline{\mathcal{D}(0, r, 1)} \backslash\left(V_{1} \cup V_{2}\right)$. One easily finds $n_{3}$ so that $\left|f_{n}(z)\right|,\left|g_{n}(z)\right|<M_{2}+1$ on $\overline{\mathcal{D}(0, r, 1)} \backslash\left(V_{1} \cup V_{2}\right)$ for $n \geq n_{3}$. We set $M_{3}=M_{2}+1$. Since for $|\alpha|,|\beta|<M_{3}$ we have $\chi(\alpha, b) \geq \frac{|\alpha-\beta|}{1+M_{3}^{2}}$, we see that $f_{n} \rightarrow f$ and $g_{n} \rightarrow g$ uniformly on $\overline{\mathcal{D}(0, r, 1)} \backslash\left(V_{1} \cup V_{2}\right)$ with respect to the Eucledian metric in $\mathbb{C}=\mathbb{R}^{2}$. Thus, $f_{n}+g_{n} \rightarrow f+g$ uniformly on $\overline{\mathcal{D}(0, r, 1)} \backslash\left(V_{1} \cup V_{2}\right)$ with respect to the Eucledian metric in $\mathbb{C}=\mathbb{R}^{2}$. Since $\chi(\alpha, \beta) \leq$ $|\alpha-\beta|$, it follows that $f_{n}+g_{n} \rightarrow f+g$ uniformly on $\overline{\mathcal{D}(0, r, 1)} \backslash\left(V_{1} \cup V_{2}\right)$ with respect to $\chi$ metric. Thus, there exists $n_{4}$, so that $\sup _{z \in \overline{\mathcal{D}(0, r, 1)} \backslash\left(V_{1} \cup V_{2}\right)} \chi\left(f_{n}(z)+g_{n}(z), f(z)+g(z)\right)<\frac{\varepsilon}{3}$. We set $n_{0}=\max \left(n_{1}, n_{2}, n_{4}\right)$ and we have the result.

The limiting case $r=1$ of the previous result is quite different. 
Theorem 5.4. The uniform limits of the trigonometric polynomials $\sum_{-N}^{N} a_{n} z^{n}$ on $T=$ $\{z:|z|=1\}$ with respect to the $\chi$ metric are exactly all continuous functions $f: T \rightarrow$ $\mathbb{C} \cup\{\infty\}$.

Proof. One direction is obvious. Let $f: T \rightarrow C \cup\{\infty\}$ be a continuous function and $\varepsilon>0$. We have to find a trigonometric polynomial $Q, Q(z)=\sum_{-N}^{N} a_{n} z^{n}$, so that $\sup _{\chi} \chi(f(z), Q(z))<\varepsilon$. If $f \equiv \infty$ it suffices to choose $Q(z)=n z$ for $n$ big enough. $|z|=1$

Assume there exists $z_{0} \in T$ so that $f\left(z_{0}\right) \in \mathbb{C}$. If $f(z) \neq \infty$ for all $z \in T$, then $f: T \rightarrow \mathbb{C}$ being continuous it can be approximated by a trigonometric polynomial $Q$ so that $\sup _{z \in T}|f(z)-Q(z)|<\varepsilon$. Since $\chi(\alpha, \beta) \leq|\alpha-\beta|$, it follows $\sup _{z \in T} \chi(f(z), Q(z))<\varepsilon$.

It remains to examine the case $f\left(z_{0}\right) \in \mathbb{C}$ and $f\left(z_{1}\right)=\infty$ for some $z_{0}, z_{1} \in T$. We consider $M<+\infty$ so that the $\chi$ diameter of the set $E=\{\infty\} \cup\{z \in \mathbb{C}:|z| \geq M\}$ is less than $\frac{\varepsilon}{2}$. Then $f^{-1}\left(E^{0}\right)$ is an open subset of $T$ containing $f^{-1}(\infty)$. The compact set $f^{-1}(\infty)$ is covered by a finite number of components of $f^{-1}(E)$.

Thus, $f^{-1}(\infty) \subset\left\{e^{i \theta}: \theta \in \bigcup_{n=1}^{L}\left(\alpha_{n}, \beta_{n}\right)\right\}=V$ where $\alpha_{1}<\beta_{1}<\alpha_{2}<\beta_{2}<\cdots<$ $\alpha_{L}<\beta_{L}<\alpha_{1}+2 \pi$ and $\left|f\left(e^{i \alpha_{n}}\right)\right|=\left|f\left(e^{9 \beta_{n}}\right)\right|=M$ for all $n=1, \ldots, L$. We define a new function $g: T \rightarrow \mathbb{C}$ setting $g\left(e^{i \vartheta}\right)=f\left(e^{i \vartheta}\right)$ for $e^{i \vartheta} \in T \backslash V$. For each $n=1, \ldots, L$ we choose $g_{\mid\left\{e^{i \theta}: \theta \in\left(\alpha_{n}, \beta_{n}\right)\right\}}:\left\{e^{i \theta}: \theta \in\left(\alpha_{n}, \beta_{n}\right)\right\} \rightarrow\{z \in \mathbb{C}:|z|=M\}$ continuous so that $\lim _{\theta \rightarrow \alpha_{n}^{+}} g\left(e^{i \theta}\right)=f\left(e^{i \alpha_{n}}\right)$ and $\lim _{\theta \rightarrow \beta_{n}^{-}} g\left(e^{i \theta}\right)=f\left(e^{i \beta_{n}}\right)$. For this, it suffices to follow one of the arcs of the circle $\{z \in \mathbb{C}:|z|=M\}$ with starting point $f\left(e^{i \alpha_{n}}\right)$ and ending to $f\left(e^{i \beta_{n}}\right)$. It is easy to check that $g: T \rightarrow \mathbb{C}$ is continuous and that, by the choice of $M$ we have $\sup _{z \in T} \chi(f(z), g(z))<\frac{\varepsilon}{2}$. Now $g$ taking values on $\mathbb{C}$ and being continuous it can be approximated uniformly on $T$ by a trigonometric polynomial $Q$, with respect to the Eucledian metric on $\mathbb{C}=\mathbb{R}^{2}$. We choose $Q(z)=\sum_{-N}^{N} a_{n} z^{n}$ so that $\sup _{z \in T}|g(z)-Q(z)|<\frac{\varepsilon}{2}$. Since $\chi(\alpha, \beta) \leq|\alpha-\beta|$ it follows $\sup _{z \in T} \chi(g(z), Q(z))<\frac{\varepsilon}{2}$. Since we also have $\sup _{z \in T} \chi(f(z), g(z))<\frac{\varepsilon}{2}$ the triangular inequality implies $\sup _{z \in T}(f(z), Q(z))<\varepsilon$ and the proof is completed.

If $I$ is a compact segment or a homeomorphic image of a compact segment, $I \subset \mathbb{C}$, then the uniform on $I$ limits of the polynomials are all continuous functions $f: T \rightarrow$ $\mathbb{C} \cup\{\infty\}$. This is another particular case of the required extension of Mergelyan's Theorem. The proof is similar to that of Theorem 5.4. with the difference that we do not approximate by a trigonometric polynomial but by a polynomial. This is possible 
by the classical Mergelyan's Theorem, because $I^{0}=\emptyset$ and $I^{c}$ is connected.

Another particular case of possible extension of Mergelyan's Theorem is the following.

Let $L_{1}, L_{2}, \ldots, L_{N}$ be a finite collection of compact subsets of $\mathbb{C}$ with connected complements. We assume that for each $L_{i}, i=1, \ldots, N$ the extension of Mergelyan's Theorem is valid. We also assume that the $L_{i}$ 's, $i=1, \ldots, N$ are two by two disjoint. Then the extension of Mergelyan's Theorem is valid for $L_{1} \cup L_{2} \cup \cdots \cup L_{N}$. Indeed, if $f: L_{1} \cup \cdots \cup L_{N} \rightarrow \mathbb{C} \cup\{\infty\}$ is continuous and for every $i=1, \ldots, N$ either $f_{\mid L_{i}} \equiv \infty$ or $f\left(L_{i}^{0}\right) \subset \mathbb{C}$ and $f_{\mid L_{D}^{0}}$ holomorphic, then by assumption there exist polynomials $P_{i}$, $i=1, \ldots, N$ so that $\sup _{z \in L_{i}} \chi\left(f(z), P_{i}(z)\right)<\frac{\varepsilon}{2}$ for all $i=1, \ldots, N$. Now by the classical Mergelyan's Theorem on $L=L_{1} \cup L_{2} \cup \cdots \cup L_{N}$, there exists a polynomial $P$ so that $\sup _{z \in L_{i}}\left|P(z)-P_{i}(z)\right|<\frac{\varepsilon}{2}$. Since $\chi(\alpha, \beta) \leq|\alpha-\beta|$, we have $\sup _{z \in L_{i}} \chi\left(P(z), P(z) \mid<\frac{\varepsilon}{2}\right.$.

The triangular inequality gives the result.

So in particular the extension of Mergelyan's Theorem is valid for the union of two disjoint closed discs. What happens for the union of a sequence of pairwise disjoint closed discs converging to one point $P$ in $\mathbb{C}$ ? The difficulty is when $f(P)=\infty$. 


\section{Appendix}

In this Appendix I collect the basic facts about the metric $\chi$ we used (and proved) in this article.

Because $\chi(\alpha, \beta) \leq|\alpha-\beta|$ for all $\alpha, \beta \in \mathbb{C}$ it follows that uniform convergence with respect to the Eucledian metric in $\mathbb{C}=\mathbb{R}^{2}$ implies uniform convergence with respect to the metric $\chi$. The converse does not hold in general. However, if $f_{n} \rightarrow f$ uniformly with respect to $\chi$ on some set $E$, and there exist $M<+\infty$ so that $\left|f_{n}(z)\right| \leq M$ and $|f(z)| \leq M$ for all $z \in E$ an all $n \geq n_{0}$ for some $n_{0}$, then it follows that $f_{n} \rightarrow f$ uniformly on $E$ with respect to the Eucledian metric on $\mathbb{C}=\mathbb{R}^{2}$. The reason is that $\chi\left(f_{n}(z), f(z)\right) \geq \frac{\left|f_{n}(z)-f(z)\right|}{1+M^{2}}$.

In fact it suffices that only $|f(z)| \leq M$ on $E$ (or that only $\mid f_{n}(z) \leq M$ on $E$ for all $n \geq n_{0}$ for some $\left.n_{0}\right)$. The reason is that when $\sup _{z \in E} \chi\left(f_{n}(z), f(z)\right) \rightarrow 0$ and $|f(z)| \leq M$ on $E$, then there exists $n_{0}$ so that for $n \geq n_{0}$ we have $\left|f_{n}(z)\right| \leq M+1$ on $E$.

Another fact we have used (and proved) is that, if $\Omega$ is a domain and $f_{n}: \Omega \rightarrow \mathbb{C}$ a sequence of holomorphic functions in $\Omega$ and there exists a function $f: \Omega \rightarrow \mathbb{C} \cup\{\infty\}$ so that $\sup _{z \in \Omega} \chi\left(f_{n}(z), f(z)\right) \rightarrow 0$, then either $f \equiv \infty$ or $f(\Omega) \subset \mathbb{C}$ and $f$ is holomorphic in $\Omega$. This follows by Hurwitz Theorem.

Finally we have proven the following on an annulus $\overline{D(0, r, 1)}, 0<r<1$.

If $f_{n}: \overline{\mathcal{D}(0, r, 1)} \rightarrow \mathbb{C}$ and $g_{n}: \overline{\mathcal{D}(0, r, 1)} \rightarrow \mathbb{C}$ and $f: \overline{\mathcal{D}(0, r, 1)} \rightarrow \mathbb{C} \cup\{\infty\}$ and $g: \overline{\mathcal{D}(0, r, 1)} \rightarrow C \cup\{\infty\}$ are given. $f$ and $g$ are continuous and $f^{-1}(\infty) \subset\{z:|z|=1\}$ and $g^{-1}(\infty) \subset\{z:|z|=r\}$.

Assume $\sup _{z \in \overline{\mathcal{D}(0, r, 1)}} \chi\left(f_{n}(z), f(z)\right) \rightarrow 0$ and $\underset{z \in \overline{\mathcal{D}(0, r, 1)}}{\sup } \chi\left(g_{n}(z), g(z)\right) \rightarrow 0$.
Then $\sup _{z \in \frac{\mathcal{D}(0, r, 1)}{} \chi\left(f_{n}(z)+g_{n}(z), f(z)+g(z)\right) \rightarrow 0 \text { and } f+g \text { is well defined. }}$

I think the above facts except probably the last one are well known. May be we can find them in the works of Caratheodory, or Zalcman. The proofs may be shortened if we did references to articles proving the above facts.

Acknowledgment. I would like to thank G. Costakis for bringing to my attention reference [8] and for a helpful communication. 


\section{References}

[1] V. Nestoridis, Universal Taylor series, An. Inst. Fourier (Grenoble) 46 (1996), 1293-1306.

[2] J.-P. Kahane, Baire's Category Theorem and Trigonometric series, J. Anal. Math. vol 80 (2000), 143-182.

[3] Melas and Nestoridis, University of Taylor series as a generic property of holomorphic functions, Advances in Math. 157 (2001) 138-176.

[4] Costakis, Nestoridis and Papadoperakis, Universal Laurent series, Proc. Edinb. Math. Soc. 48 (2005) 571-583.

[5] P. Koosis, Introduction to $H_{p}$ spaces, London Math. Soc. Lecture Note Series 40, Cambridge Univ. Press, Cambridge London, N.Y. New Rochelle, Melbourne, Sydney.

[6] P. L. Duren, Theory of $H^{p}$ spaces, Acad. Press, N.Y., 1970.

[7] K. Hoffman, Banach Spaces of Analytic Functions, M.I.T., Prentice-Hall, Inc. Englewood Cliffs, N.J.

[8] D. Stegenga and K. Stephenson, Generic covering properties for spaces of Analytic Functions, Pacific J. Math. Vol. 119, No 6, (1985), 227-243.

University of Athens

Department of Mathematics

15784 Panepistemiopolis

Athens

GREECE

e-mail: vnestor@math.uoa.gr 